\title{
Star-forming Clumps in Local Luminous Infrared Galaxies
}

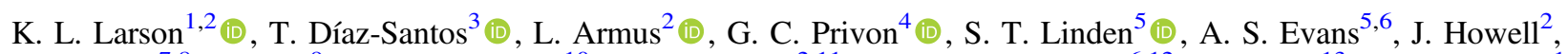

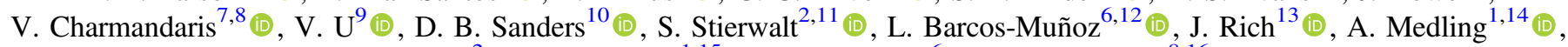

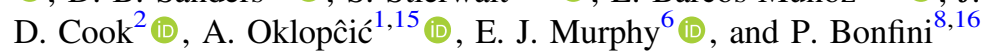 \\ ${ }^{1}$ Department of Astronomy, California Institute of Technology, 1200 E. California Blvd., Pasadena, CA 91125, USA \\ ${ }^{2}$ IPAC, California Institute of Technology, 1200 E. California Blvd., Pasadena, CA 91125, USA \\ ${ }^{3}$ Núcleo de Astronomía de la Facultad de Ingeniería, Universidad Diego Portales, Av. Ejército Libertador 441, Santiago, Chile \\ ${ }^{4}$ Department of Astronomy, University of Florida, 211 Bryant Space Sciences Center, Gainesville, FL 32611, USA \\ ${ }^{5}$ Department of Astronomy, University of Virginia, Charlottesville, VA 22904, USA \\ ${ }^{6}$ National Radio Astronomy Observatory, 520 Edgemont Rd., Charlottesville, VA 22903, USA \\ ${ }^{7}$ Department of Physics, University of Crete, GR-71003, Heraklion, Greece
${ }^{8}$ Institute of Astrophysics, Foundation for Research and Technology-Hellas, Heraklion, GR-70013, Greece \\ ${ }^{9}$ Department of Physics and Astronomy, 4129 Frederick Reines Hall, University of California, Irvine, CA 92697, USA \\ ${ }^{10}$ Institute for Astronomy, University of Hawaii, 2680 Woodlawn Dr., Honolulu, HI 96822, USA \\ ${ }^{11}$ Department of Physics, Occidental College, 1600 Campus Rd., Los Angeles, CA 90041, USA \\ ${ }^{12}$ Joint ALMA Observatory, Alonso de Córdova 3107, Vitacura, Santiago, Chile \\ ${ }^{13}$ Carnegie Observatories, 813 Santa Barbara St., Pasadena, CA 91101, USA \\ ${ }^{14}$ Ritter Astrophysical Research Center, University of Toledo, Toledo, OH 43606, USA \\ ${ }^{15}$ Institute for Theory and Computation, Harvard-Smithsonian Center for Astrophysics, 60 Garden St., MS-51, Cambridge, MA 02138, USA \\ ${ }^{16}$ Institute for Astronomy, Astrophysics, Space Applications \& Remote Sensing, National Observatory of Athens, P. Penteli, GR-15236 Athens, Greece \\ Received 2019 March 12; revised 2019 October 25; accepted 2019 November 18; published 2020 January 13
}

\begin{abstract}
We present HST narrowband near-infrared imaging of $\mathrm{Pa} \alpha$ and $\mathrm{Pa} \beta$ emission of 48 local luminous infrared galaxies (LIRGs) from the Great Observatories All-Sky LIRG Survey. These data allow us to measure the properties of 810 spatially resolved star-forming regions (59 nuclei and 751 extranuclear clumps) and directly compare their properties to those found in both local and high-redshift star-forming galaxies. We find that in LIRGs the star-forming clumps have radii ranging from $\sim 90$ to $900 \mathrm{pc}$ and star formation rates (SFRs) of $\sim 1 \times 10^{-3}$ to $10 M_{\odot} \mathrm{yr}^{-1}$, with median values for extranuclear clumps of $170 \mathrm{pc}$ and $0.03 M_{\odot} \mathrm{yr}^{-1}$. The detected star-forming clumps are young, with a median stellar age of $8.7 \mathrm{Myr}$, and have a median stellar mass of $5 \times 10^{5}$ $M_{\odot}$. The SFRs span the range of those found in normal local star-forming galaxies to those found in high-redshift star-forming galaxies at $z=1-3$. The luminosity function of the LIRG clumps has a flatter slope than found in lower-luminosity, star-forming galaxies, indicating a relative excess of luminous star-forming clumps. In order to predict the possible range of star-forming histories and gas fractions, we compare the star-forming clumps to those measured in the MassiveFIRE high-resolution cosmological simulation. The star-forming clumps in MassiveFIRE cover the same range of SFRs and sizes found in the local LIRGs and have total gas fractions that extend from 10\% to $90 \%$. If local LIRGs are similar to these simulated galaxies, we expect that future observations with ALMA will find a large range of gas fractions, and corresponding star formation efficiencies, among the star-forming clumps in LIRGs.
\end{abstract}

Unified Astronomy Thesaurus concepts: Interacting galaxies (802); Star forming regions (1565); Luminous infrared galaxies (946)

Supporting material: machine-readable table

\section{Introduction}

Star formation processes are central to understanding the buildup of mass in galaxies over time. Star formation occurs over a large range in physical scale, from parsec-sized molecular clouds to large kiloparsec-sized star-forming regions in the disks and nuclei of galaxies (e.g., Kennicutt \& Evans 2012). Connecting star formation over these vastly different scales is difficult but essential to understanding fundamental observable signatures of galaxy evolution, such as the main sequence of star-forming galaxies and the role of mergers (Daddi et al. 2007; Elbaz et al. 2007, 2011, Rodighiero et al. 2011). Additionally, resolving star-forming regions and linking emission from young stars to the physical conditions and gas supply is critical to understanding the ubiquity and genesis of the Kennicutt-Schmidt (KS) law (Schmidt 1959;
Kennicutt 1998) and the variation of star formation efficiency across galaxies and galaxy types.

Recently, a number of surveys have found that high- $z$ starforming galaxies tend to display turbulent, clumpy disks with extreme star-forming clumps, having masses of $\sim 10^{8}-10^{9} M_{\odot}$ and sizes of $0.5-5 \mathrm{kpc}$ (Elmegreen et al. 2004, 2009; Genzel et al. 2008; Daddi et al. 2010; Livermore et al. 2015). These clumps are $\sim 100 \times$ larger than typical giant molecular clouds locally. Most galaxies studied in detail at high $z$ host a few large, kiloparsec-sized star-forming clumps. In contrast, local star-forming galaxies contain hundreds of smaller H II regions (e.g., Cowie et al. 1995; Wisnioski et al. 2012; Cook et al. 2016), suggesting a fundamental shift in the way stars form in galaxies at these early epochs.

One possible interpretation is that these large star-forming clumps are a result of higher gas fractions and increased star formation efficiency (Tacconi et al. 2008; Jones et al. 2010; 
Mieda et al. 2016). Simulations by Tamburello et al. (2015) support this hypothesis, showing that simulated galaxies with high gas fractions are more likely to have large star-forming clumps than galaxies with lower gas fractions. Furthermore, these massive clumps appear to be present in both interacting and noninteracting high- $z$ galaxies.

Turbulence and high gas fractions have both been invoked to explain the extreme properties of star-forming clumps in high-z galaxies (Dekel et al. 2009; Förster Schreiber et al. 2011; Soto et al. 2017). However, published studies often have limited spatial resolution and sensitivity, dropping normal star-forming regions well below the detection limits and making it difficult to resolve sources on the scales of traditional H II regions. The true luminosity function (LF) and size distributions of starforming regions in high- $z$ galaxies are therefore still highly uncertain.

Recent progress has been made using strong gravitational lensing of $z=1-3$ galaxies, which has allowed for clump studies at resolutions down to $100 \mathrm{pc}$. Livermore et al. (2012) found that the average star formation rate (SFR) density $\left(\mathrm{SFR} \mathrm{kpc}^{2}\right)$ of star-forming clumps in lensed, star-forming galaxies increases with redshift. Other authors have studied the effects of resolution by smoothing intrinsically high-resolution data to lower resolution to mimic the effects of observing high$z$ galaxies without the benefit of lensing. Fisher et al. (2017), in a study of $z \sim 0.1$ galaxies, found that the size of the detected clumps grew and the number of clumps fell as a larger fraction of smaller clumps got incorporated in the beam. Cava et al. (2018) directly tested the effects of resolution on clump properties by studying multiple lensed images of the same $z=0.4385$ galaxy at different magnifications. The different magnifications allow them to study the same galaxy at resolutions from 30 to $300 \mathrm{pc}$ and show that lower resolution, not surprisingly, causes the clumps' size and masses to be systematically overestimated.

These studies illustrate the need to study star-forming clumps in actively star-forming galaxies at high spatial resolution and with enough sensitivity to probe the faint end of the LF. It is also important to be able to separate clumps from multiple nuclei and disk clumps from those in and around the nuclei, especially in galaxies undergoing interactions and mergers, which provide the trigger for the enhanced star formation, since in these systems circumnuclear clumps can be quite complex and the differences between nuclei and luminous star-forming clumps can become blurred.

Luminous infrared galaxies (LIRGs), with thermal IR [8-1000 $\mu \mathrm{m}$ ] dust emission in excess of $10^{11} L_{\odot}$, are an ideal laboratory for studying resolved star formation in the local universe. The bolometric luminosity of LIRGs is dominated by massive bursts of star formation, showing also a wide range of contributions from active galactic nuclei (AGNs; see Petric et al. 2011; Stierwalt et al. 2013, 2014). Multiwavelength observations have shown that local LIRGs are a mixture of single-disk galaxies, interacting systems, and advanced mergers, exhibiting enhanced SFRs and AGN activity compared to less luminous and noninteracting galaxies (see Sanders \& Mirabel 1996; Stierwalt et al. 2014; Larson et al. 2016). By comparing star formation in LIRGs to normal (noninteracting and $L_{\mathrm{IR}}<10^{11}$ ) low-redshift galaxies, high-redshift galaxies, and sophisticated hydrodynamical simulations, we can better understand how global galaxy properties and environment influence star formation on smaller scales.
Because LIRGs are, by their very nature, dusty, it is important to study star formation in these systems in the infrared, where obscuration is minimized while still achieving high spatial resolution. In star-forming galaxies the hydrogen recombination emission lines are used extensively as SFR estimators, since their fluxes provide a straightforward measure of the number of ionizing photons produced by massive O/B stars. However, the central kiloparsec of LIRGs is often heavily enshrouded and can be subject to extremely large visual extinctions, $A_{V}>10$ (García-Marín et al. 2009; Piqueras López et al. 2013; Stierwalt et al. 2013), making the optical $\mathrm{H} \alpha$ and $\mathrm{H} \beta$ lines poor tracers of the star formation (Armus et al. 1989). The extinction throughout the galaxy is much less severe in the near-infrared $\left(1.6 \mu \mathrm{m} \sim 0.2 \times \mathrm{A}_{V}\right)$, and lines such as $\mathrm{Pa} \alpha, \mathrm{Pa} \beta$, or $\mathrm{Br} \gamma$ can provide a much more complete picture of the obscured star formation.

Integral field spectra observations on large, ground-based telescopes can be used to simultaneously measure the distribution and luminosity of star-forming clumps, as well as an estimate of the extinction from ratios of the Paschen and Bracket recombination lines (e.g., U et al. 2019), but currently these studies have extremely limited fields of view. For example, in a detailed study of 17 (U)LIRGs using SINFONI on the Very Large Telescope (VLT), Piqueras López et al. (2016) found $\mathrm{Pa} \alpha$ luminosities and SFRs for some clumps that rival those seen at high redshift in the inner $8^{\prime \prime} \times 8^{\prime \prime}$ (typically $3 \mathrm{kpc}$ ) of their galaxies. Deep, high-resolution, wide field of view (FOV) studies are better suited to measuring the star formation across entire merging systems as a function of merger stage and disk properties, and for comparison to galaxies at high redshift.

Here we present a Hubble Space Telescope (HST) nearinfrared, narrowband imaging study of the properties of 810 star-forming clumps in a sample of 48 local LIRGs. The sample galaxies cover a range of merger stages, from undisturbed noninteracting galaxies to highly disturbed late-stage mergers. The spatial resolution of $H S T$ enables us to quantify the fraction of star formation occurring in clumps versus faint, diffuse emission and to measure the size, luminosities, and spatial distribution of star-forming clumps on subkiloparsec scales.

In this paper we present the global properties of star-forming clumps in local LIRGs, measuring the SFRs, sizes, ages, and masses of star-forming clumps via the high-resolution $\mathrm{Pa} \alpha$ and $\mathrm{Pa} \beta$ HST images. In a follow-up paper (Paper II), we will investigate how the clump properties change with individual galaxy properties like the global SFR, sSFR, and merger stage and as a function of radial distance in each galaxy. In Section 4.1 we measure the LF of the star-forming clumps and compare the slope of the clump LF in the Great Observatories All-sky LIRG Survey (GOALS) to that found in local galaxies by Cook et al. (2016). In Section 4.2 we investigate the size and SFR of the clumps and compare them to both high-redshift $(1<z<4)$ star-forming clumps from Livermore et al. (2012, 2015) and star-forming clumps in $z=0$ normal (noninteracting and $L_{\mathrm{IR}}<10^{11}$ ) galaxies in the SINGS sample. Finally, in Section 4.3 we compare our GOALS clumps to starforming regions in galaxies in MassiveFIRE, a high-resolution cosmological hydrodynamical simulation.

\section{Sample Selection and Observations}

GOALS (Armus et al. 2009) is a complete galaxy sample that comprises the 201 LIRG systems $(z<0.088)$ included in 
the IRAS Revised Bright Galaxy Sample (Sanders et al. 2003) and is aimed at measuring the physical properties of local LIRGs across the electromagnetic spectrum using a broad suite of ground- and space-based observatories.

Here we present and analyze $\mathrm{Pa} \alpha$ and $\mathrm{Pa} \beta H S T$ images of a sample of 48 LIRGs in GOALS with $L_{\mathrm{IR}}$ values that range from $10^{11.2}$ to $10^{12.3}$. The Pa $\alpha$ data (27 systems) are HST archival images from PID 10169. These galaxies were selected to have a redshift range of $0.0093 \leqslant z \leqslant 0.0174$ so that the $\mathrm{Pa} \alpha$ emission line lies within the HST NICMOS F190N narrowband filter (Alonso-Herrero et al. 2006). Galaxies in the $\mathrm{Pa} \beta$ sample were selected to have redshifts between $0.0225 \leqslant z \leqslant 0.0352$ so that the $\mathrm{Pa} \beta$ emission line lies within the HST WFC 3 F132N narrowband filter. Since most of the galaxies in the $\mathrm{Pa} \alpha$ sample were isolated galaxies and early-stage mergers, mid- to latestage mergers were chosen for the expanded $\mathrm{Pa} \beta$ sample. The $\mathrm{Pa} \beta$ sample is therefore not a flux-limited sample, as it does not contain all the galaxies in the redshift range that could be observed. The combination of the $\mathrm{Pa} \alpha$ and $\mathrm{Pa} \beta$ samples contains 12 noninteracting galaxies, 17 early-stage mergers, and 17 mid- and late-stage mergers, providing a full representation of the entire interaction sequence. When all the galaxies in pairs are counted, there are a total of 59 individual galaxies in the sample.

\subsection{Observations}

The $\mathrm{Pa} \beta$ observations were taken with the HST WFC3 camera (Project ID: 13690; PI: T. Díaz-Santos), which has a plate scale of 0 " 12 pixel $^{-1}$ using the F130N and F132N narrowband filters and the F110W broadband filter. The total integration times were $120 \mathrm{~s}$ for each broadband filter and about $1100 \mathrm{~s}$ (19 minutes) for each narrowband filter. The F130N filter is used to measure the underlying continuum emission next to the line. The 2' FOV of WFC3-IR allows for galaxies in a pair to be observed simultaneously. Each observation was again divided into three individual exposures with offsets of 5 pixels.

The archival $\mathrm{Pa} \alpha$ observations were taken with the HST NIC2 camera, which has a plate scale of 0 ". 076 pixel $^{-1}$ and a 19!" 2 FOV using the F187N and F190N narrowband filters. This FOV allows for detection of $\mathrm{P} \alpha$ emission up to a radial distance of $\sim 5 \mathrm{kpc}$ from the galaxy nucleus. From our $\mathrm{Pa} \beta$ data, which cover a much larger FOV, we find that most of the Paschen emission is indeed contained within $5 \mathrm{kpc}$ from the galaxy nucleus. For objects with redshifts between 0.0093 and 0.0174 the $\mathrm{Pa} \alpha$ emission line, at $1.87 \mu \mathrm{m}$, is captured with the F190N filter, while the adjacent filter, F187N, is used for continuum subtraction. The typical total integration times were 900-950 s for each narrowband filter. Each observation was divided into three individual exposures with offsets of 5 pixels. For details on the data reduction of the $\mathrm{Pa} \alpha$ observations we refer the reader to Alonso-Herrero et al. (2006). Observational details are given for every galaxy in our combined $\mathrm{Pa} \alpha$ and $\mathrm{Pa} \beta$ sample in Table 1.

\section{Data Reduction and Analysis}

The WFC3-IR images from Cycle 23 were reduced using the standard $H S T$ pipeline, which includes drizzling and stacking of the images. The pipeline also corrects for instrumental effects by removing bad pixels, subtracting the dark, flatfielding, and performing gain conversion. The Pyraf Tweakreg and drizzelpac packages were then used to align and co-add the individual exposures in each filter. Tweakreg was used to find the offsets for all images by using the position of bright stars visible in all filters. All of the individual images for a given object were then aligned to a common reference image using these offsets while ensuring that the co-added images for each filter would also be aligned with each other. Finally, the Pyraf drizzelpac package was used to correct for geometric distortions, remove cosmic rays, and co-add the individual images into a fully reduced mosaic image for each filter.

\subsection{Matching Physical Resolution}

In order to have a consistent comparison of star-forming clumps between all the galaxies in the combined sample, we rebinned and smoothed all images to a common physical resolution of $91 \mathrm{pc}$ pixel $^{-1}$, which corresponds to the resolution of the most distant galaxy in our sample. To create the continuum-subtracted $\mathrm{Pa} \alpha$ and $\mathrm{Pa} \beta$ line images, the narrowband images (F190N and F187N for $\mathrm{Pa} \alpha$ and F132N and $\mathrm{F} 130 \mathrm{~N}$ for $\mathrm{Pa} \beta$ ) were convolved with the corresponding line's point-spread function (PSF) to create images with matched pixel scales and PSFs before subtraction. This allowed for a final clean continuum-subtracted line image at a common pixel scale for all galaxies. Figure 1 shows the broadband F110W $H S T$ images and the continuum-subtracted $\mathrm{Pa} \beta$ line images for both NGC 6786 and NGC 6090. Depending on the position of the line in the narrowband filter, there can be significant flux loss (Alonso-Herrero et al. 2000). We model a Gaussian emission-line width of $200 \mathrm{~km} \mathrm{~s}^{-1}$ to estimate and correct for the flux loss due to the position of the line in the narrowband filter. In general, the position of the Paschen line is well contained in the narrowband filter and has less than a 5\% flux loss. One exception is NGC 5257-8, which does have significant estimated flux loss of $70 \%$ owing to the position of $\mathrm{Pa} \beta$ in the narrowband filter.

\subsection{Clump Analysis Procedure}

Our ability to compare star-forming clump properties to high- $z$ and high-resolution simulations depends on being able to consistently detect star-forming clumps with a wide range of luminosities and sizes. In order to detect the clumps and measure their properties, we use the continuum-subtracted line images and the python code astrodendro. ${ }^{17}$ Astrodendro computes a dendrogram, which is a branching tree of hierarchical structure, of an image. This procedure compares each possible clump to the local background, successfully identifying clumps down to the lowest detectable flux limit (Figure 2).

There are three important parameters in the astrodendro code that affect the identification of clumps. The first is the minimum size of the region. Since the FWHM of the data is $\sim 1$ pixel, we set a minimum size of 3 pixels to be accepted as a clump. The 3-pixel minimum size maximizes the detection of the smallest clumps while avoiding blending of clumps. Then, a minimum threshold above the general background level is also required, which we set at $5 \sigma$ above the general sky level of the image. This ensures that we have a resolved clump that is well above any background noise or diffuse emission. Lastly, a minimum significance for the substructure is set that determines when a

\footnotetext{
${ }^{17}$ http://www.dendrograms.org
} 
Table 1

Observations

\begin{tabular}{|c|c|c|c|c|}
\hline Galaxy Name & IRAS Name & $z$ & Filter & PIDs \\
\hline NGC 0023 & IRAS F00073+2538 & 0.015231 & F187N, F190N & 10169 \\
\hline MCG -02-01-051 & IRAS F00163-1039 & 0.027152 & F130N, F132N & 13690 \\
\hline MCG + 12-02-001 & IRASF $00506+7248$ & 0.015698 & F187N, F190N & 10169 \\
\hline IC 0214 & IRAS F02114+0456 & 0.030224 & F130N, F132N & 13690 \\
\hline UGC 1845 & IRASF $02208+4744$ & 0.015607 & F187N, F190N & 10169 \\
\hline$\ldots \ldots$ & IRAS F02437+2122 & 0.023306 & F130N, F132N & 13690 \\
\hline NGC 1614 & IRASF 04315-0840 & 0.015938 & F187N, F190N & 10169 \\
\hline$\ldots \ldots$ & IRAS $05129+5128$ & 0.027432 & F130N, F132N & 13690 \\
\hline UGC 3351 & IRASF $05414+5840$ & 0.01486 & F187N, F190N & 10169 \\
\hline NGC 2369 & IRASF 07160-6215 & 0.010807 & F187N, F190N & 10169 \\
\hline NGC 2388 & IRASF $07256+3355$ & 0.01379 & F187N, F190N & 10169 \\
\hline CGCG 058-009 & IRASF $07329+1149$ & 0.016255 & F187N, F190N & 10169 \\
\hline ESO 320-G030 & IRASF $11506-3851$ & 0.010781 & F187N, F190N & 10169 \\
\hline NGC 4922 & IRAS F12590+2934 & 0.023589 & F130N, F132N & 13690 \\
\hline MCG +02-33-098 & IRASF 12596-1529 & 0.015921 & F187N, F190N & 10169 \\
\hline$\ldots \ldots$ & IRAS $13120-5453$ & 0.030761 & F130N, F132N & 13690 \\
\hline UGC 08387 & IRAS F13182+3424 & 0.02330 & F130N, F132N & 13690 \\
\hline IC 0860 & IRASF $13126+2453$ & 0.011164 & F187N, F190N & 10169 \\
\hline NGC 5135 & IRASF 13229-2934 & 0.013693 & F187N, F190N & 10169 \\
\hline NGC 5734 & IRASF 14423-2039 & 0.013746 & F187N, F190N & 10169 \\
\hline NGC 5257-8 & IRAS F13373+0105 & 0.0225 & F130N, F132N & 13690 \\
\hline NGC 5331 & IRAS F13497+0220 & 0.033043 & F130N, F132N & 13690 \\
\hline IC $4518 \mathrm{E} / \mathrm{W}$ & IRASF $14544-4255$ & 0.015728 & F187N, F190N & 10169 \\
\hline VV 340a & IRAS F14547+2449 & 0.033669 & F130N, F132N & 13690 \\
\hline ESO 099-G004 & IRAS 15206-6256 & 0.029284 & F130N, F132N & 13690 \\
\hline NGC 6701 & IRASF $18425+6036$ & 0.013226 & F187N, F190N & 10169 \\
\hline NGC 6786 & IRAS F19120+7320 & 0.025017 & F130N, F132N & 13690 \\
\hline NGC 7130 & IRASF $21453-3511$ & 0.016151 & F187N, F190N & 10169 \\
\hline IC 5179 & IRASF 22132-3705 & 0.011415 & F187N, F190N & 10169 \\
\hline NGC 7469 & IRASF $23007+0836$ & 0.016317 & F187N, F190N & 10169 \\
\hline NGC 7591 & IRASF $23157+0618$ & 0.016531 & F187N, F190N & 10169 \\
\hline ..... & IRAS $23436+5257$ & 0.034134 & F130N, F132N & 13690 \\
\hline NGC 7771 & IRASF $23488+1949$ & 0.014267 & F187N, F190N & 10169 \\
\hline
\end{tabular}

Note. The $\mathrm{P} \alpha$ and $\mathrm{P} \beta$ galaxy sample. Column (1): galaxy name. Column (2): IRAS galaxy name. Column (3): redshift. Column (4): HST narrowband filters. Column (5): HST Proposal ID number.

substructure is brighter than the local background. The minimum significance is the difference in surface density required between a structure and any substructures for a substructure to be retained. When a substructure is retained, it is shown as a new branch in the dendrogram tree in Figure 2. We set the minimum significance to $1 \sigma$ above the local background. We then take the upper branch of the dendrogram tree (red lines in the left panel of Figure 2) as our final clump regions. Astrodendro also detects any bright artifacts left in the images from bright stars or noise. Therefore, the clumps are all confirmed by visual inspection, and any sources not associated with the galaxy are removed. A detailed comparison of astrodendro to the other commonly used CLUMPFIND algorithm (Williams et al. 1994) for identifying and measuring clump properties in our sample LIRGs is given in the Appendix.

Both the continuum emission and the line emission must be accurately measured in order to determine the ages and masses of all the clumps. The same clump regions found with astrodendro on the $\operatorname{Pa} \alpha$ and $\mathrm{Pa} \beta$ line images were used on the narrowband continuum images. We subtract the local background, determined by the region surrounding the clump and not including any other clumps, to measure the clump 

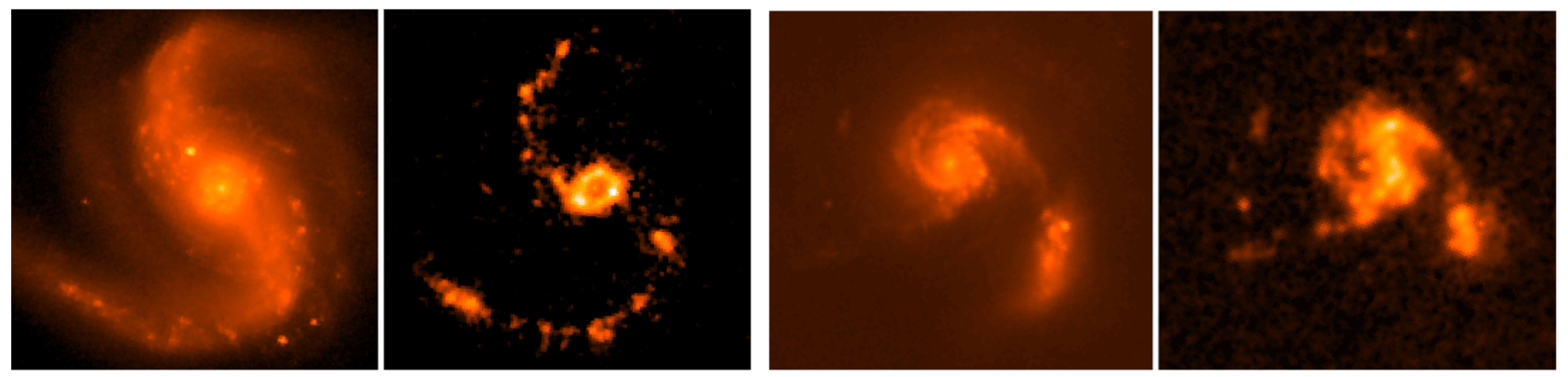

Figure 1. Left pair: HST/WFC3 F110W (left) and continuum-subtracted Pa $\beta$ image (right) of NGC 6786. Right pair: HST/WFC3 F110W (left) and continuumsubtracted $\mathrm{Pa} \beta$ image (right) of NGC 6090. Clumps of star formation and diffuse emission are visible in both NGC 6786 and the late-stage merger NGC 6090. In particular, NGC 6786 has a ring of star formation around the nucleus, while NGC 6090 contains a bright extranuclear starburst between the two interacting nuclei.

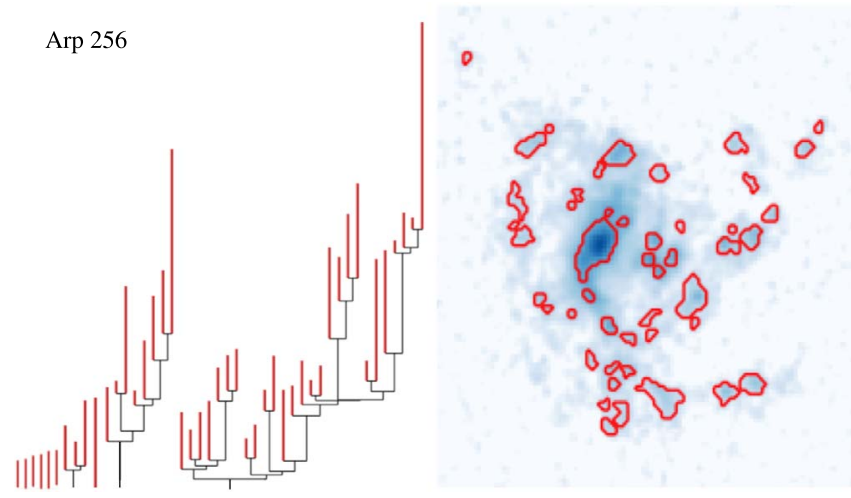

Figure 2. Astrodendro is used to select individual star-forming regions in our galaxies, shown here for Arp 256. Left: dendrogram for Arp 256. The dendrogram procedure searches for all structures brighter than the local background and finds clumps down to the lowest detectable fluxes. Right: corresponding regions for the bright structures found with astrodendro (shown in red) for Arp 256.

fluxes. The local background regions were visually inspected for each clump in line and continuum images in order to avoid hot spots or other continuum features that could cause an oversubtraction of the clump line and/or continuum fluxes. If they were contaminated by continuum features, alternative clean background regions near the source were selected. Local background subtraction is incredibly important to ensure that the resulting SFRs, ages, and masses are for the clump and not contaminated by underlying stellar populations (Guo et al. 2018).

We determine the errors in the clump sizes using a Monte Carlo method. Astrodendro is rerun 1000 times randomly adjusting the flux of each pixel in the image while sampling from a Poisson distribution. The standard deviation of the size is determined from the measured radii of each region from the 1000 iterations. This allows us to characterize the accuracy of the region's size based on the noise in the image.

\section{Results}

In total, we find 810 star-forming clumps: 59 nuclear clumps and 751 extranuclear clumps. The median number of extranuclear clumps we find per LIRG is 15.6. The number of clumps per LIRG varies from a merging galaxy dominated by only two bright nuclei and no extranuclear clumps to a galaxy pair with 95 extranuclear clumps.

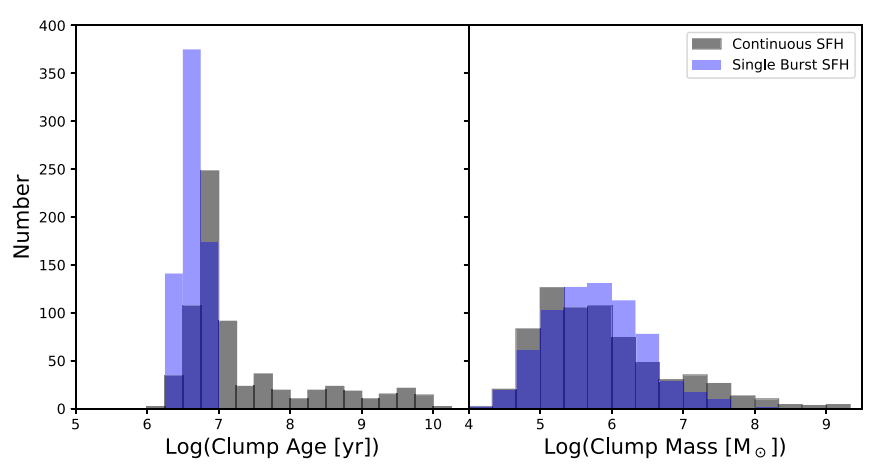

Figure 3. Distribution of the measured ages and masses for extranuclear starforming clumps found in our study. The results for a continuous SFH model are shown in black, and those for the single-burst SFH model are shown in blue.

\subsection{Ages and Masses}

The ages and masses of the clumps were computed by comparing the measured line and continuum fluxes of the clumps to Starburst99 models (Leitherer et al. 1999). We estimated the $\operatorname{Pa} \alpha$ and $\mathrm{Pa} \beta$ equivalent widths of every clump and matched them to the corresponding equivalent widths of the model spectra that were calculated in time steps of $0.1 \mathrm{Myr}$ to determine the clump ages. We compared to both an instantaneous burst single stellar population model and a continuous star formation model with a Kroupa initial mass function (IMF; Kroupa 2001) and solar metallicity for our clumps. The masses of the clumps were calculated with the background-subtracted continuum luminosities measured from the narrowband filters $(\mathrm{F} 130 \mathrm{~N}$ or $\mathrm{F} 187 \mathrm{~N})$ and the estimated mass-to-light ratio from the model $M\left(M_{\odot}\right)=L_{\text {cont-filter }} \times(M / L)$. The mass-to-light ratio varies with the age of the stellar population and was estimated for each clump at the fitted age.

Figure 3 shows the distributions of ages and masses for extranuclear star-forming clumps. The instantaneous burst model gave a narrow range of ages from $2 \times 10^{6} \mathrm{yr}$ to $6.8 \times 10^{6} \mathrm{yr}$ with a median age of $4(+0.8,-1) \times 10^{6} \mathrm{yr}$. All uncertainties are given at the $68 \%$ confidence levels. The continuous burst has a wider range of stellar ages from $1.5 \times 10^{6}$ yr to $1.6 \times 10^{10} \mathrm{yr}$, with a median age of $8.7(+6$, $-2) \times 10^{6} \mathrm{yr}$. We found that both the instantaneous burst and continuous star formation models give masses of extranuclear clumps that range from $10^{4}$ to $10^{9} M_{\odot}$ with a similar median clump mass of $\sim 5 \times 10^{5} M_{\odot}$. The median and range of clump ages and masses using both the instantaneous burst and continuous star formation models for the extranuclear clumps are given in Table 2. Since the nuclei can be contaminated by 
Table 2

Average Extranuclear Clump Properties

\begin{tabular}{lcccc}
\hline \hline Parameter & Min & Max & Median & Mean \\
\hline Log(Age)-Cont. & 6.17 & 10.21 & $6.94(+0.24,-0.10)$ & 7.33 \\
Log(Age)-Inst. & 6.29 & 6.84 & $6.65(+0.70,-0.11)$ & 6.63 \\
Log(Mass)-Cont. & 4.12 & 9.22 & $5.69(+0.44,-0.38)$ & 5.87 \\
Log(Mass)-Inst. & 4.14 & 8.31 & $5.73(+0.36,-0.29)$ & 5.77 \\
SFR & 0.002 & 4.43 & $0.03(+0.03,-0.01)$ & 0.088 \\
Radius & 89 & 678 & $171(+47,-26)$ & 199 \\
$\Sigma_{\text {SFR }}$ & 0.045 & 14.86 & $0.31(+0.18,-0.10)$ & 0.55 \\
\hline
\end{tabular}

Note. The minimum, maximum, median, and mean values for extranuclear clump properties are given. The $68 \%$ confidence levels are denoted for the medians in the table. The ages and masses are given for both the continuous SFH and single-burst SFH. Row 1: age $\log ([\mathrm{yr}])$ using the continuous SFH; row 2: age $\log ([\mathrm{yr}])$ using the single-burst SFH; row 3: mass $\log \left(\left[M_{\odot}\right]\right)$ using continuous SFH; row 4: mass $\log \left(\left[M_{\odot}\right]\right)$ using single-burst SFH; row 5: SFR $\left[M_{\odot} \mathrm{yr}^{-1}\right]$; row 6 : effective radius [pc], where the minimum radius is set by the minimum allowed clump size of 3 pixels; row 7 : SFR surface density $\left[M_{\odot} \mathrm{yr}^{-1}\right.$ $\left.\mathrm{kpc}^{-2}\right]$.

AGNs and may be effected by higher levels of extinction, we exclude the nuclei from Figure 3 and Table 2.

Even with careful background subtraction, we were unable to calculate accurate ages and masses for 45 extranuclear clumps. These clumps have large errors on the continuum measurements, which lead to errors in the age estimate that are as large as the age. They have very little continuum detected and therefore large errors that do not allow for accurate estimates of the ages and masses. We therefore exclude these clumps from our discussion of ages, masses, and SFRs. Further discussion of ages, masses, and SFRs of extranuclear clumps will only include the 706 clumps. A further 21 of these extranuclear clumps have unconstrained ages owing to equivalent widths that are outside of the range predicted by the continuous star formation history (SFH) model. These clumps are clearly identified in both line and continuum emission and are therefore included in the future discussion of SFRs.

\subsection{Star Formation Rates and Sizes}

The clumps we find using the astrodendro technique span a large range of luminosities and sizes as shown in Figure 4. The area of the clump is determined by the number of pixels in the region, and the effective radius is defined as $\mathrm{r}=\sqrt{A / \pi}$. To allow for an equal comparison of both $\mathrm{Pa} \alpha$ and $\mathrm{Pa} \beta$ images and other $\mathrm{H} \alpha$ clump studies, we convert all clump luminosities to SFRs. We use a conversion factor determined from Starburst 99 stellar population models assuming a continuous star formation, Kroupa IMF, and stellar metallicity at the appropriate age for each clump. This conversion factor uses the "case B" line intensity ratios and assumes no extinction. While extinction from dust obscuration is much less in the near-infrared than in the optical for U/LIRGs, it can still affect the nuclei of the galaxies and decreases quickly in the extranuclear regions (Díaz-Santos et al. 2011). Previous studies using VLT/ SINFONI data have shown that local $(z<0.02)$ LIRGs have subkiloparsec clumpy dust structures. These regions have a wide range of $A_{\mathrm{v}}$ from 1 to $20 \mathrm{mag}$ and an average $A_{\mathrm{v}}=5.3$ mag within their $3 \times 3 \mathrm{kpc}$ FOV (Piqueras López et al. 2013). We therefore cannot apply a single galactic $A_{\mathrm{v}}$ to our starforming clumps since the dust obscuration is extremely patchy

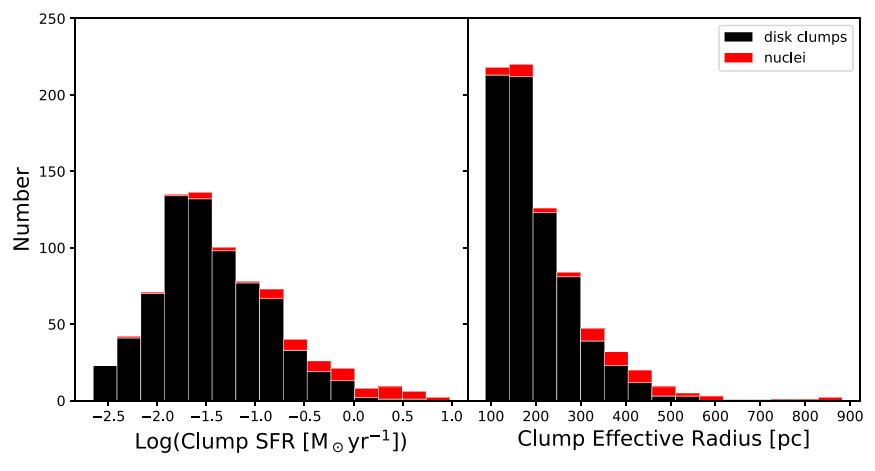

Figure 4. Distribution of the measured SFR and sizes for all regions. Extranuclear clumps are shown in black, while nuclear clumps are shown in red. The Paschen line luminosities were converted to SFRs based on a continuous SFH model.

and each galaxy presents different spatial clump distributions. In a study of star clusters in GOALS galaxies, Linden et al. (2019) estimated the extinction to individual extranuclear clusters using far-UV (FUV), $B$, and $I$ photometry. Thirty-two of our star-forming clumps distributed over six galaxies overlap with their sample of detected extranuclear star clusters. The estimated $A_{\mathrm{v}}$ for these extranuclear regions ranges from 0.2 to 1.8 mag with an average $A_{\mathrm{v}}=1$, which implies an $A_{\mathrm{NIR}}$ $=0.1-0.2 \mathrm{mag}$. Therefore, while extinction corrections can be important for some extremely dusty clumps, the extinction is highly spatially variable and can be fairly low outside of the circumnuclear regions. Our nuclear clumps likely have the largest corrections, but these are unknown. The fluxes and derived SFRs of the nuclear clumps are therefore, effectively, lower limits.

Due to large continuum measurements and accordingly low equivalent width in some of the nuclei, 20 of the 59 nuclear clumps have equivalent widths that are below the range predicted by the continuous SFH models. For the 20 nuclei and the 21 extranuclear clumps with equivalent widths not covered in the continuous SFH models, we cannot determine an age. The clump SFRs are therefore calculated using the asymptotic calibration value from the Starburst99 continuous SF model at $10^{8} \mathrm{yr}\left(\mathrm{SFR} / L_{\mathrm{H} \alpha}=5.37 \times 10^{-42}\left[M_{\odot} \mathrm{yr}^{-1}\right] /\left[\mathrm{erg} \mathrm{s}^{-1}\right]\right)$. While the Starburst99 continuous SF model provides a variable luminosity to SFR calibration with age, the calibration starts to asymptote to a constant value at an age of $5 \times 10^{6} \mathrm{yr}$ and reaches a constant value by $10^{8} \mathrm{yr}$. The asymptotic calibration reached for our Starburst99 models is the same calibration determined by Murphy et al. (2011).

In Figures 4 and 5, we see that the clump SFRs span a wide range from $2 \times 10^{-3}$ to $4.4 M_{\odot} \mathrm{yr}^{-1}$ with nuclear clumps that have SFRs of $5 \times 10^{-3}$ to $9.6 M_{\odot} \mathrm{yr}^{-1}$. We find clump radii that range from our minimum resolvable size of $89 \mathrm{pc}$ up to 883 pc, with extranuclear clumps reaching sizes of $678 \mathrm{pc}$ and a median of $171(+47,-26)$ pc.

We provide the measurements of all clumps with determined SFRs in our $\mathrm{Pa} \alpha$ and $\mathrm{Pa} \beta$ sample in Table 3. The clumps are identified by their host galaxy and ID number (GalaxyName_c\#). Data for nuclei in each galaxy are also given and identified by the host galaxy name and nuclei number (GalaxyName_n\#). The estimated age and mass of each clump are given, assuming a continuous SFH and solar metallicity, as well as the clump SFR and effective radius.

The SFR surface density $\left(\Sigma_{\mathrm{SFR}}\right)$ of the extranuclear GOALS clumps ranges from 0.045 to $14.86 M_{\odot} \mathrm{yr}^{-1} \mathrm{kpc}^{-2}$. Star- 


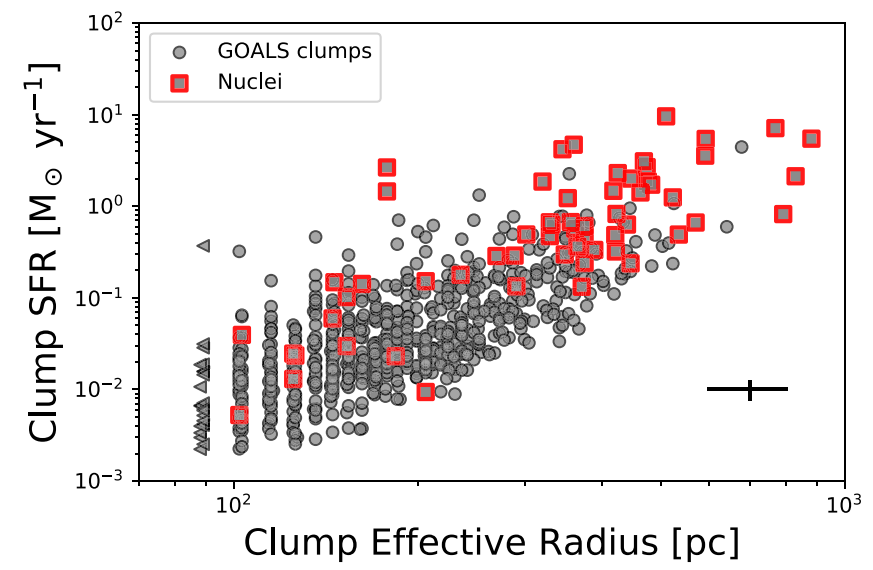

Figure 5. Individual clump SFRs as a function of size for all star-forming regions found in the GOALS $\mathrm{Pa} \alpha$ and $\mathrm{Pa} \beta$ sample. Some of the brightest and largest clumps coincide with the nuclei of the galaxies, and these are marked with a red box. The sizes of the smallest clumps are upper limits and are indicated with a left-pointing triangle. Average error bars for the data are given in the lower right corner.

forming regions with $\Sigma_{\mathrm{SFR}}>1 M_{\odot} \mathrm{yr}^{-1} \mathrm{kpc}^{-2}$ are often referred to as "starbursting" since extranuclear star-forming regions in normal spiral galaxies are found to have $\Sigma_{\text {SFR }}$ below this cutoff (Maragkoudakis et al. 2017; Nguyen-Luong et al. 2016; Alonso-Herrero et al. 2000; Kennicutt 1998). Furthermore, Cosens et al. (2018) have also found a break in the sizeluminosity scaling relation at a $\Sigma_{\mathrm{SFR}}=1 M_{\odot} \mathrm{yr}^{-1} \mathrm{kpc}^{-1}$. Figure 6 shows how the majority of the clumps lie below $\Sigma_{\mathrm{SFR}}=1 \quad M_{\odot} \mathrm{yr}^{-1} \mathrm{kpc}^{-2}$, with $13 \%$ of the extranuclear clumps in the "starburst" region. The median $\Sigma_{\mathrm{SFR}}$ of extranuclear clumps is $0.31(+0.19,-0.10) M_{\odot} \mathrm{yr}^{-1} \mathrm{kpc}^{-2}$, while nuclear clumps have a higher median $\Sigma_{\mathrm{SFR}}=1.4(+0.8$, -0.5) $M_{\odot} \mathrm{yr}^{-1} \mathrm{kpc}^{-2}$ and are more likely to be starbursting, with $64 \%$ of the nuclear clumps in the "starburst" region. The median and range of clump SFRs and $\Sigma_{\text {SFR }}$ values for the extranuclear clumps are given in Table 2. Piqueras López et al. (2016) observed a sample of 17 LIRGs and ULIRGs with the VLT/SINFONI integral field unit and found a wider range of SFR surface densities for clumps in the range of $0.1-60 \Sigma_{\mathrm{SFR}}$, although these data were corrected for extinction (from the $\operatorname{Br} \gamma$ and $\operatorname{Br} \delta$ ratios). We converted the Piqueras López et al. (2016) results from Salpeter to Kroupa IMF to be comparable to the results presented in this paper. The wide range of SFR surface densities found by Piqueras López et al. (2016) is due to their applied extinction correction. The clumps with the largest $\Sigma_{\mathrm{SFR}}$ in their study are the ones with the largest inferred extinction, and these tend to be in the nuclei, making up a small fraction of the distribution. Therefore, the median observed and extinction-corrected $\Sigma_{\mathrm{SFR}}$ for the ULIRGs in the Piqueras López et al. (2016) sample are $\Sigma_{\mathrm{SFR}}^{\mathrm{obs}}=0.11 \mathrm{M}_{\odot} \mathrm{yr}^{-1}$ $\mathrm{kpc}^{-2}$ and $\Sigma_{\mathrm{SFR}}^{\mathrm{corr}}=0.16 \mathrm{M}_{\odot} \mathrm{yr}^{-1} \mathrm{kpc}^{-2}$, similar to the median extranuclear $\Sigma_{\mathrm{SFR}}$ found in our sample of $\Sigma_{\mathrm{SFR}}=0.31$.

Even though our galaxy sample is selected to be infrared luminous, the majority of our extranuclear star-forming clumps have $\Sigma_{\text {SFR }}$ at the upper end of $\Sigma_{\text {SFR }}$ values that are found in normal spiral galaxies, with $13 \%$ of the clumps in the starburst region.

The total SFR, derived from the HST data, includes the clumpy and diffuse narrowband emission. For each galaxy, this is determined by converting the Paschen luminosity to $\mathrm{H} \alpha$ luminosity assuming the "case B" recombination and then converting the $\mathrm{H} \alpha$ luminosity to SFR using the calibration from Murphy et al. (2011) (SFR $/ L_{\mathrm{H} \alpha}=5.37 \times 10^{-42}$ $\left.\left[M_{\odot} \mathrm{yr}^{-1}\right] /\left[\mathrm{erg} \mathrm{s}^{-1}\right]\right)$. This calibration assumes a Kroupa IMF with continuous SFR and solar metallicity integrated over a timescale of $100 \mathrm{Myr}$. We use the Murphy et al. (2011) calibration for obtaining the total SFR of the galaxies since we are averaging over a range of sizes and ages of star-forming clumps and diffuse emission. Figure 7 shows the fraction of the total SFR, as derived from the infrared emission, that is detected in each galaxy in the narrowband HST images. The total infrared SFR of the galaxies is calculated by converting the total infrared luminosity of the galaxies, $L_{\mathrm{IR}}$, to an SFR (Murphy et al. 2011).

There is a large range in the percentage of the total SFR, as traced in the far-infrared (FIR), recovered in our Paschen data (Figure 7). The median ratio of $\mathrm{P} \beta$ or $\mathrm{P} \alpha$ to total FIR-derived star formation in our sample is $9 \%$. The galaxy with the lowest detected Paschen emission is IC 0860 , with only $0.14 \%$ of the total infrared emission detected, and is not shown in Figure 7. This galaxy has no extranuclear clumps, and all detected emission is from the nucleus. This source must be heavily enshrouded even in the near-infrared.

Figure 7 shows the percentage of the Paschen emission for each galaxy observed in clumps. The percentage of the total Paschen emission contained in extranuclear clumps is $0 \%$ to $65 \%$, with a median of $14.7 \%$. When the nuclei are included, $10 \%-100 \%$ of the Paschen emission is contained in clumps, with the remaining emission in diffuse emission. The nuclear regions can contain a significant fraction of the detected Paschen emission even without extinction correction. Some galaxies, such as NGC 5258 and NGC 6786, are dominated by extranuclear star formation with no detectable nuclear emission in $\mathrm{Pa} \beta$, whereas other galaxies, such as IRAS F02437+2122, IRAS $23436+5257$, and NGC 7591 , are dominated by strong nuclear emission with little or no extranuclear star-forming clumps.

\section{Discussion}

\subsection{Luminosity Function}

The LF of star-forming clumps in local galaxies can be well approximated by a power law with a slope of $\alpha=-2$ (e.g., Zhang \& Fall 1999; de Grijs et al. 2003; Chandar et al. 2015; Cook et al. 2016). Local LIRGs cover an SFR of $20<$ SFR $\left[M_{\odot} \mathrm{yr}^{-1}\right]<200$, thus making up the high-LF end.

Previous studies have shown that different binning methods can change the measured slope of the LF (Maíz Apellániz \& Úbeda 2005, Cook et al. 2016). While the equal luminositysized bins is the most commonly used method, Maíz Apellániz \& Úbeda (2005) showed that this caused systematic biases at the brightest luminosity bins owing to low number statistics. Therefore, we chose to use variable bin sizes where each bin contains an equal number of sources.

While we do not have enough clumps per galaxy to do individual LFs for each galaxy in our sample, we can create a combined function of clumps from all GOALS galaxies. Since we are comparing data from a combined $\mathrm{P} \alpha$ and $\mathrm{P} \beta$ sample, we create an SFR function using the SFRs of the clumps as defined previously in Section 4.2. Only extranuclear clumps are included in the SFR function.

We fit the data in two ways to determine the slope. First, we determine the slope by Markov Chain Monte Carlo (MCMC) 
Table 3

Properties of Star-forming Clumps in GOALS Galaxies

\begin{tabular}{|c|c|c|c|c|c|c|c|c|}
\hline $\begin{array}{l}\text { Clump } \\
\text { Name }\end{array}$ & $\begin{array}{l}\text { Radius } \\
\quad(\mathrm{pc})\end{array}$ & $\begin{array}{l}\text { Err } \\
(\mathrm{pc})\end{array}$ & $\begin{array}{c}\mathrm{SFR} \\
\left(M_{\odot} \mathrm{yr}^{-1}\right)\end{array}$ & $\begin{array}{c}\text { Err } \\
\left(M_{\odot} \mathrm{yr}^{-1}\right)\end{array}$ & $\begin{array}{l}\text { Age } \\
(\mathrm{yr})\end{array}$ & $\begin{array}{l}\text { Err } \\
(\mathrm{yr})\end{array}$ & $\begin{array}{l}\text { Mass } \\
\left(M_{\odot}\right)\end{array}$ & $\begin{array}{c}\text { Err } \\
\left(M_{\odot}\right)\end{array}$ \\
\hline MCG +02-20-003_n1 & 269 & 24 & 0.286 & 0.009 & $\mathrm{NaN}$ & $\mathrm{NaN}$ & $\mathrm{NaN}$ & $\mathrm{NaN}$ \\
\hline MCG +02-20-003_c1 & 254 & 80 & 0.026 & 0.007 & $6.1 \mathrm{e}+08$ & $4.5 \mathrm{e}+08$ & $1.5 \mathrm{e}+07$ & $1.2 \mathrm{e}+07$ \\
\hline MCG +02-20-003_c2 & 103 & 21 & 0.006 & 0.001 & $3.6 \mathrm{e}+09$ & $1.5 \mathrm{e}+09$ & $1.8 \mathrm{e}+07$ & $6.7 e+06$ \\
\hline MCG +02-20-003_c3 & 187 & 29 & 0.008 & 0.002 & $3.8 \mathrm{e}+09$ & $1.8 \mathrm{e}+09$ & $2.6 e+07$ & $1.2 \mathrm{e}+07$ \\
\hline MCG +02-20-003_c4 & 127 & 24 & 0.003 & 0.001 & $1.8 \mathrm{e}+09$ & $1.1 \mathrm{e}+09$ & $4.9 \mathrm{e}+06$ & $2.6 e+06$ \\
\hline MCG +02-20-003_c5 & 116 & 22 & 0.010 & 0.001 & $1.3 e+07$ & $3.0 \mathrm{e}+06$ & $1.2 \mathrm{e}+05$ & $4.8 \mathrm{e}+04$ \\
\hline MCG +02-20-003_c6 & 226 & 19 & 0.048 & 0.005 & $1.0 \mathrm{e}+07$ & $2.0 \mathrm{e}+06$ & $4.9 \mathrm{e}+05$ & $2.8 \mathrm{e}+05$ \\
\hline
\end{tabular}

Note. Properties of star-forming clumps found in GOALS galaxies. Column (1): source name. Column (2): effective radius of the clump in pc calculated from the clump area. Upper limits are indicated with a negative value. Column (3): radius error [pc]. Column (4): clump SFR in $M_{\odot} \mathrm{yr}^{-1}$ calculated from the Pa $\alpha$ or Pa $\beta$ flux. Column (5): clump SFR error. Column (6): clump age [yr] calculated from the estimated equivalent width. Column (7): clump age error [yr]. Column (8): clump mass in $M_{\odot}$. Column (9): clump mass error.

(This table is available in its entirety in machine-readable form.)

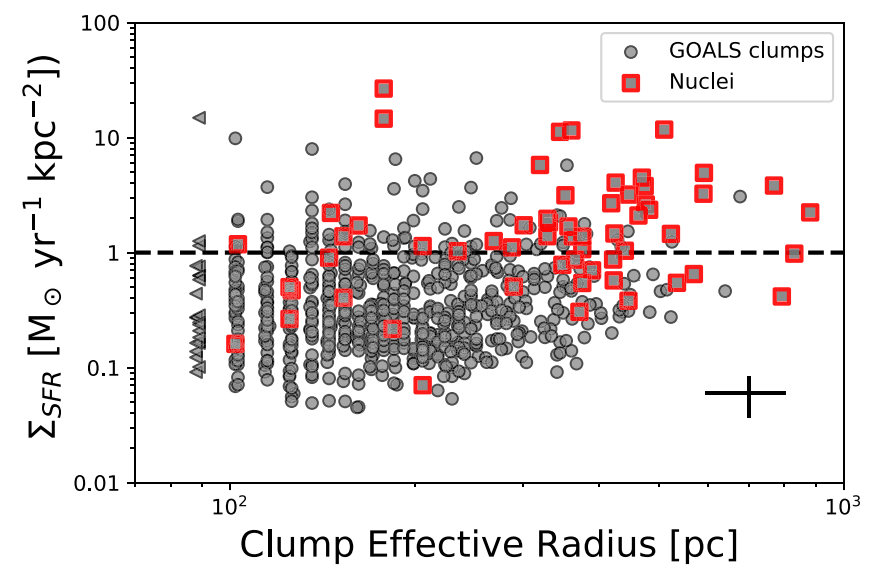

Figure 6. SFR density of the $\mathrm{Pa} \alpha$ and $\mathrm{Pa} \beta$ clumps as a function of clump size. The nuclear clumps are marked with a red box, and, as expected, they typically have larger sizes and higher luminosities than the star-forming clumps in the disks. The sizes of the smallest clumps are upper limits and are indicated with a left-pointing triangle. Average error bars for the data are given in the lower right corner.

modeling of the SFR function as a power law plus an exponential cutoff. This simultaneously fits the slope and the incompleteness cutoff point, giving a slope of $\alpha=-1.65 \pm 0.06$ and an SFR cutoff of $0.015 \pm 0.002 M_{\odot}$ $\mathrm{yr}^{-1}$ as shown in the left panel of Figure 8. The flattening of the number of sources at lower SFRs is due to an incompleteness in the data at the faint end.

Second, we fit a single power law to only the bright end of the SFR function, where the data are complete. We also bin the data using an equal number per bin and determine the error on each bin by bootstrap resampling so we can compare directly to the results from Cook et al. (2016). The SFR cutoff for the fit is determined by the peak of the SFR histogram, at $\log$ (SFR cutoff) $=-1.7$. We find that the combined SFR function of clumps from all GOALS galaxies has a bright-end slope of $\alpha=1.49 \pm 0.06$.

A study of UV star-forming regions in nearby galaxies by Cook et al. (2016) investigated the dependence of the clump LF on the galaxy SFRs $\left(10^{-3}<\operatorname{SFR}\left[M_{\odot} \mathrm{yr}^{-1}\right]<3\right)$. They calculated the SFRs of clumps in their sample by converting dusted-corrected FUV fluxes to SFRs using the Murphy et al. (2011) prescription and Kroupa IMF. Cook et al. (2016) found that galaxies with higher SFRs tend to have flatter clump LF slopes. They then derived a combined LF of clumps from 134 galaxies and found a bright-end slope of $\alpha=-1.93$. The galaxies in the Cook sample range in distance from $\sim 1$ to $\sim 10.5 \mathrm{Mpc}$, corresponding to regions with diameters of $\sim 24-250$ pc. In Figure 8, we also fit only the local normal galaxies having distances larger than $5.8 \mathrm{Mpc}$ (equivalent to a lower limit on the clump resolution of about $70 \mathrm{pc}$ ) to match the GOALS sample, and we find a bright-end slope of $\alpha=-1.90 \pm 0.03$, still within the limits of what was initially found with the full sample. This demonstrates that the flattening of the slope that we see in the GOALS sample is not solely due to resolution effects.

The combined SFR function slope from the normal galaxies is steeper than that found in the GOALS galaxies as can be seen in Figure 8. While both studies suffer from incompleteness at the faint end, it is clear that the bright-end slopes are significantly different, suggesting an overabundance of bright, SF extranuclear clumps in LIRGs. Furthermore, the variable nature of extinction in LIRGs and ULIRGs means that some of the clumps in our sample could have $A_{\mathrm{v}}$ as high as 10-20 even if on average the $A_{\mathrm{v}}$ is much lower (Piqueras López et al. 2013 , 2016). While we cannot correct for extinction directly in our data, if there was a significant effect, it would imply many more luminous clumps and further flatten the slope of the LF, increasing the difference between the local, normal galaxies and GOALS galaxies.

\subsection{Luminosity and Size}

While the range of sizes of star-forming clumps in local LIRGs is similar to that in local, normal $\left(L_{\mathrm{IR}}<10^{11}\right)$ galaxies, the LIRG clumps have much larger SFR (see Figure 9). Many are comparable to star-forming clumps seen in high-redshift galaxies. A significant fraction of high- $z$ galaxies $(1<z<3)$ have turbulent, clumpy, disks with clump masses of $\sim 10^{8}-10^{9}$ $M_{\odot}$ and sizes of 0.5-5 kpc (e.g., Elmegreen et al. 2004, 2009; Daddi et al. 2010). Studying UV star-forming clumps in galaxies in the Hubble Ultra Deep Field at redshifts in the range of $0.5<z<1.5$, Soto et al. (2017) found an average clump SFR of $0.014 M_{\odot} \mathrm{yr}^{-1}$ and an average clump radius of $0.9 \mathrm{kpc}$, with some extending up to $2 \mathrm{kpc}$. The current interpretation is that these large clumps with high SFR have higher gas fractions and increased star formation efficiency (Tacconi et al. 2008; Jones et al. 2010). 

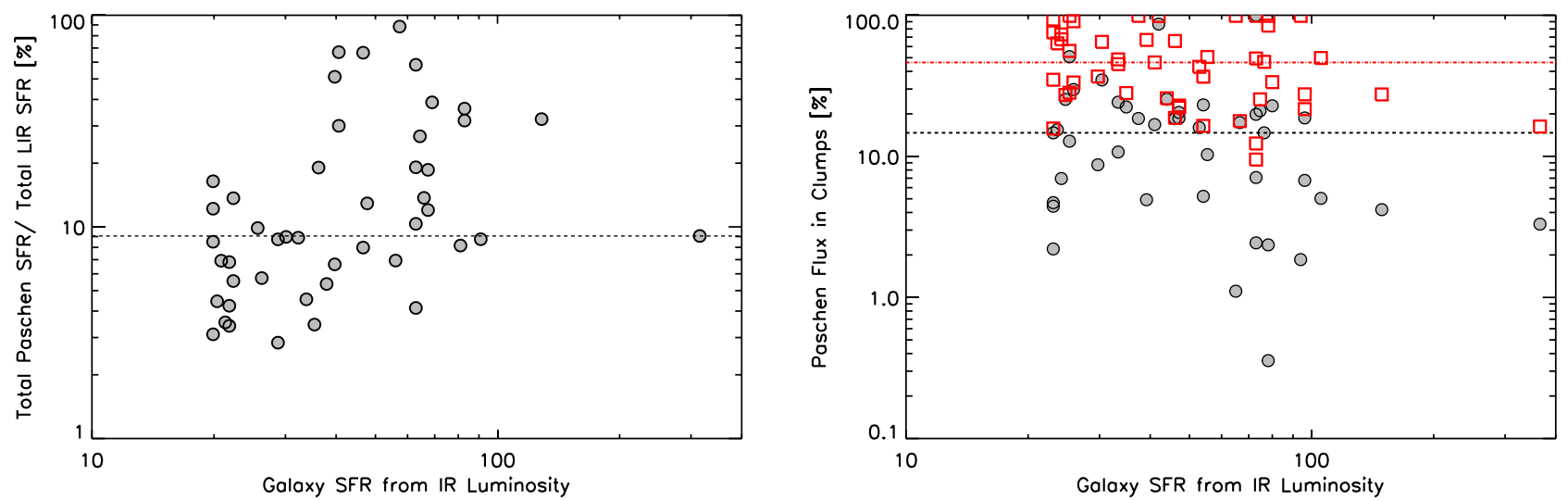

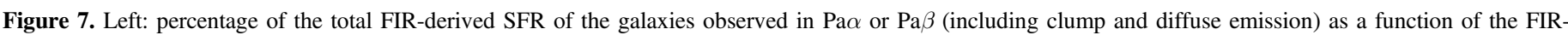

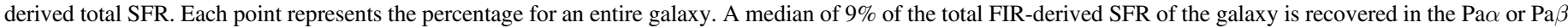

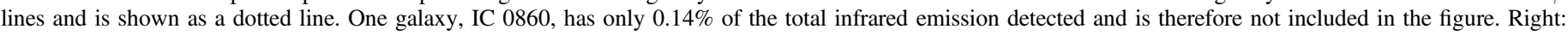

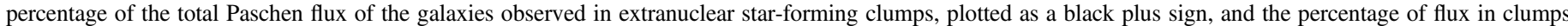

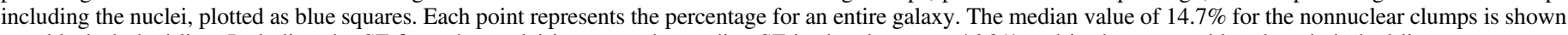
as a black dashed line. Including the SF from the nuclei increases the median SF in the clumps to $46.3 \%$ and is shown as a blue dotted-dashed line.

However, most high-redshift clump studies can only reach down to resolutions of $\sim 0.5-1 \mathrm{kpc}$, which is larger than most of the resolved clumps in the GOALS sample. Lensed galaxies provide a way to study high-redshift, clumpy star formation on scales of $100 \mathrm{pc}$ (Livermore et al. 2012, 2015). We can therefore directly compare our GOALS star-forming clumps to those found in the lensed galaxy samples.

The SFRs of GOALS clumps $\left(10^{-3}\right.$ to $\left.10\left[M_{\odot} \mathrm{yr}^{-1}\right]\right)$ span the range from SINGS $z=0$ galaxies to those found in $z=1-3$ lensed galaxies, thus bridging the gap between the local universe and the high- $z$ universe when comparing samples at similar resolutions. While local LIRGs have smaller fractions of high-luminosity clumps than galaxies at $z>2$, they provide compelling evidence that the physical conditions driving extreme star formation at high $z$ can be found in merging starburst galaxies at low redshift. We note that clumps measured in high-redshift galaxies without the benefit of lensing would lie in the upper right corner of Figure 9 with sizes of $1-5 \mathrm{kpc}$ and SFRs in the range of $\sim 1-30 M_{\odot} \mathrm{yr}^{-1}$ (Genzel et al. 2011; Swinbank et al. 2012; Wisnioski et al. 2012; Soto et al. 2017; Guo et al. 2018).

\subsection{Star-forming Clumps in High-resolution Cosmological Hydrodynamical Simulations}

Simulations of high- $z$ systems have recently been used to predict the distribution in sizes, luminosities, and lifetimes of the gas clumps in terms of the evolution of gas-rich disks at $z \sim 2$ (Oklopčić et al. 2016). It is natural, therefore, to compare our GOALS results to these models to see if they are similar and, if so, whether or not we might gain a better understanding of the nature and fate of the luminous star-forming clumps we see in GOALS.

The MassiveFIRE (Feedback in Realistic Environments) simulation has 8 times smaller mass resolution $\left(m=3.3 \times 10^{-4} M_{\odot}\right)$ than FIRE and simulates galaxies at $z>1.7$ (Feldmann et al. 2016). The high mass resolution obtained in MassiveFIRE resolves down to an SFR density of $0.07 M_{\odot} \mathrm{yr}^{-1} \mathrm{kpc}^{-2}$ and allows for a direct comparison to the observed star-forming clumps in GOALS. We have used the same software suite (astrodendro) to identify star-forming clumps in the MassiveFIRE simulation. Using astrodendro on the MassiveFIRE simulations resolves SF clumps down to a size of $126 \mathrm{pc}$ effective radius, allowing for a relevant comparison to our GOALS SF clumps. Figure 10 shows the SFR versus size for both the GOALS and MassiveFIRE starforming clumps. The clumps in MassiveFIRE have a similar range of SFRs $\left(4 \times 10^{-3}\right.$ to $\left.6.45 M_{\odot} \mathrm{yr}^{-1}\right)$ and sizes $(126 \mathrm{pc}-$ $1 \mathrm{kpc})$ to the GOALS star-forming clumps. Since the FIRE simulations model both the stars and gas, we can use these results to estimate basic properties (e.g., the star formation efficiencies) of the star-forming clumps in GOALS.

The models predict star-forming clumps with a wide range of total (atomic plus molecular) gas fractions from $\sim 10 \%$ to $\sim 90 \%$ gas. Higher star formation clumps have lower gas fractions. Most of the GOALS clumps that overlap in SFR-size space with MassiveFIRE clumps have intermediate to high clump gas fractions $(>50 \%)$, while clumps with the highest SFRs, and lowest gas fractions, do appear rare in the GOALS data. The MassiveFIRE clumps have a median star formation efficiency of $3.5 \times 10^{-9} \mathrm{yr}^{-1}$, almost an order of magnitude higher than the kiloparsec-scale star formation efficiency found in local spiral galaxies of $4.3 \times 10^{-10} \mathrm{yr}^{-1}$ (Leroy et al. 2008). Similarly high star formation efficiency has been observed for individual $250 \mathrm{pc}$ regions in one galaxy from our sample, IC 4687, which has both ALMA CO (2-1) and HST Pa $\alpha$ data (Pereira-Santaella et al. 2016).

A pilot study with ALMA is currently underway to directly measure the resolved molecular gas content on sizes comparable to our star-forming clumps. These ALMA observations of the GOALS sources will allow us to test for possible correlations with gas content and directly measure the star formation efficiencies of these clumps. In our next paper we will also investigate whether galaxy properties, such as the merger stage, are driving the SFRs in our clumps.

\section{Conclusions}

We use narrowband HST imaging to target $\mathrm{Pa} \alpha$ and $\mathrm{Pa} \beta$ emission and trace the star formation in 48 local LFGs. The LIRGs cover a range of merger stages from single isolated galaxies to galaxy pairs. Therefore, there are a total of 57 individual galaxies included in the sample.

1. A total of 810 star-forming clumps are identified in our sample of 48 LIRGs, 751 of which are extranuclear 

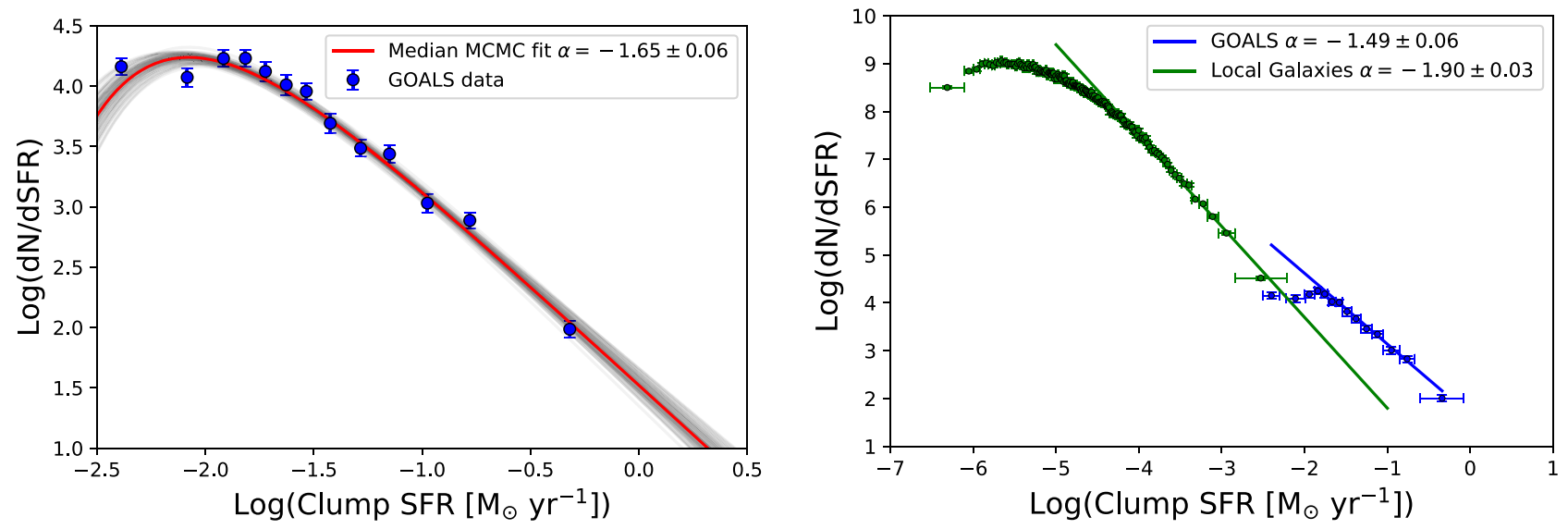

Figure 8. Left: composite SFR function for GOALS extranuclear star-forming regions. The function is modeled by a single power law plus a low-SFR exponential cutoff that gives a slope of $\alpha=-1.65 \pm 0.06$ and a $\log (\mathrm{SFR})$ cutoff of $-1.82(+.04,-.05) M_{\odot} \mathrm{yr}^{-1}$. A random selection of MCMC-derived SFR functions are overplotted in gray. Right: composite SFR functions for UV star-forming regions from the galaxies in Cook et al. (2016), shown in green, and the extranuclear starforming regions from GOALS galaxies, in blue. Both functions are fit at the bright end, where they are most complete. The GOALS sample shows a shallower slope $(-1.49 \pm 0.06)$ than local, normal, nonmerging galaxies $(-1.90 \pm 0.03)$. This may be due to the presence of larger numbers of bright, dense clumps in the GOALS galaxies.

clumps and 59 of which are nuclei. The number of clumps per galaxy ranges from two bright nuclear regions with no extranuclear clumps to a galaxy pair that has 95 extranuclear clumps. The median number of extranuclear clumps detected per LIRG is 15.6.

2. Star-forming clumps are resolved in our sample galaxies with effective radii of $89-880 \mathrm{pc}$ and SFRs of extranuclear clumps from $2 \times 10^{-3}$ to $4.4 M_{\odot} \mathrm{yr}^{-1}$. The median age and mass of star-forming clumps are $9 \times 10^{6}$ yr and $\sim 5 \times 10^{5} M_{\odot}$, respectively.

The sizes and SFRs of the clumps in GOALS galaxies span the range between the regions found in local normal galaxies and those seen at higher redshifts $(z=1-3)$. Large and luminous star-forming clumps, similar to those seen at high redshift, are found in the local universe in LIRGs.

3. Star-forming clumps in LIRGs, while exhibiting overall luminosities one to two orders of magnitude higher than star-forming clumps in most local galaxies, also have a shallower clump LF slope. The GOALS sample shows a shallower clump LF slope $(-1.49 \pm 0.06)$ than that found for star-forming regions in local, normal, nonmerging galaxies $(-1.90 \pm 0.0)$. This suggests an overabundance of luminous star-forming clumps compared to most local galaxies.

4. Star-forming clumps in local LIRGs span a similar range in SFR and size to those in high-resolution, hydrodynamic simulations (MassiveFIRE) of evolving spiral galaxies at $z \sim 2$. If the clumps modeled by the MassiveFIRE simulations are similar to those found in GOALS, this might imply high gas fractions $(>50 \%)$ and extremely large star formation efficiencies in many GOALS star-forming clumps.

With upcoming HST and ALMA observations, we will expand this sample to include galaxies with higher molecular gas fractions (above 25\%) and study the molecular gas contents of individual clumps to place them on common star formation law relations to determine whether the physical conditions in the luminous clumps are inducing exceptionally high star formation efficiencies.
K.L.L. would like to thank the anonymous referee for their useful comments, as well as P. Hopkins, J. Lee, and Y. Guo for insightful discussions that all greatly improved this paper. K.L. L. was supported by NASA through grants HST-GO13690.002-A and HST-GO-15241.002-A from the Space Telescope Science Institute, which is operated by the Association of Universities for Research in Astronomy, Inc., under NASA contract NAS5-26555. T.D-S. acknowledges support from ALMA-CONICYT project 31130005 and FONDECYT regular project 1151239. G.C.P. acknowledges support from the University of Florida. S.T.L. was supported by the NRAO Grote Reber Dissertation Fellowship. A.S.E. was supported by NSF grant AST 1109475 and by NASA through grants HSTGO1-0592.01-A, HST-GO1-1196.01-A, and HST-GO1-3364 from the Space Telescope Science Institute, which is operated by the Association of Universities for Research in Astronomy, Inc., under NASA contract NAS5-26555. V.U. acknowledges funding support from the University of California Chancellors Postdoctoral Fellowship and NSF grant AST-1412693. Support for AMM is provided by NASA through Hubble Fellowship grant HST-HF2-51377 awarded by the Space Telescope Science Institute, which is operated by the Association of Universities for Research in Astronomy, Inc., for NASA, under contract NAS5-26555. This research has made use of the NASA/IPAC Extragalactic Database (NED), which is operated by the Jet Propulsion Laboratory, California Institute of Technology, under contract with the National Aeronautics and Space Administration. This research also used astrodendro, a Python package to compute dendrograms of Astronomical data (http://www.dendrograms.org/).

\section{Appendix \\ Comparison of Astrodendro and CLUMPFIND Routines}

Since the high- and low- $z$ studies do not use a common algorithm to identify and measure star-forming clumps, it is important to compare how the selection might affect the measured distribution of properties. While we have used astrodendro to identify clumps in our GOALS sources, many of the high- $z$ studies use CLUMPFIND (Williams et al. 1994). In order to test how these two algorithms might deliver different 


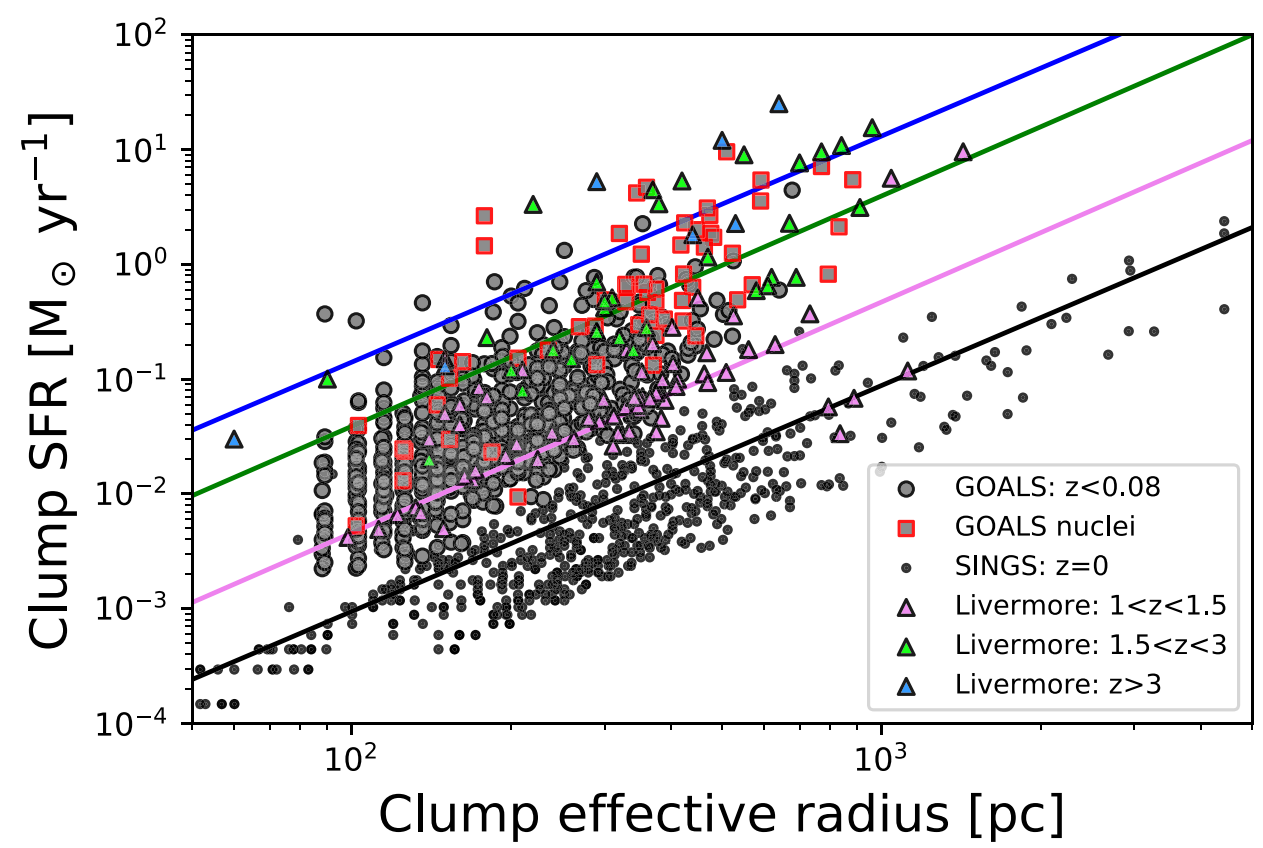

Figure 9. Clump SFR as a function of size for GOALS galaxies (gray) for both nuclear and extranuclear clumps, where the nuclear clumps are marked with a red box, compared to clumps from Livermore's 2012 and 2015 lensed sample (triangles) and local $z=0$ SINGS galaxies (black circles). Livermore's lensed clump sample is divided into three redshift bins of $1.0<z<1.5$ in purple, $1.5<z<3$ in green, and $z>3$ in blue. The lines are constant surface brightness fits to the four redshift bins, not including the GOALS SF clumps. Livermore found clumps of higher surface brightness for galaxies at higher redshift.

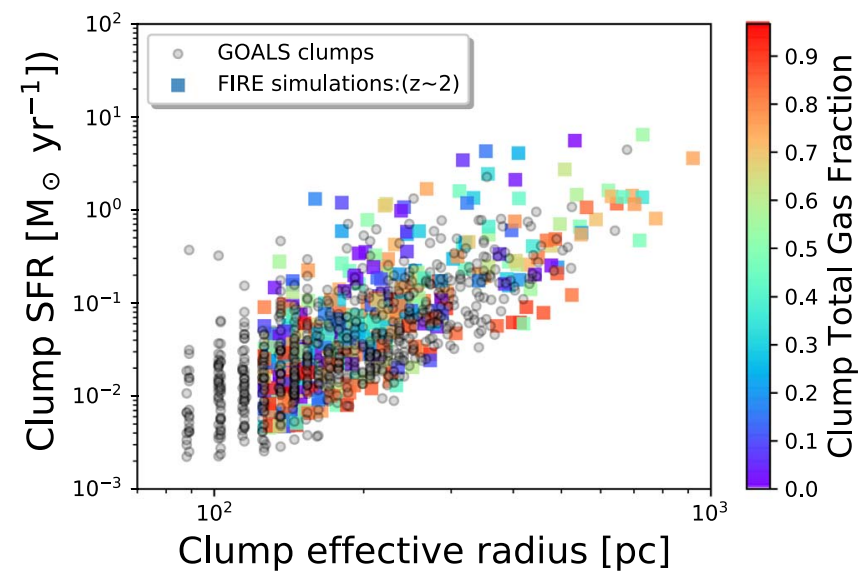

Figure 10. Comparison of extranuclear GOALS SF clumps (gray circles) and star-forming clumps identified in MassiveFIRE simulation (colored squares). The color of the MassiveFIRE symbols corresponds to the estimated total (atomic plus molecular) gas fraction of the clump. Blue symbols have gas fractions of $\sim 0.10$, and red symbols have gas fractions of $\sim 0.90$.

results, we have run CLUMPFIND on 10 of our GOALS galaxies for a direct comparison. When this is done, we find that while there is no systematic offset in SFR or radius for all the clumps, CLUMPFIND does not effectively recover the full range of SFR at a given clump radius. At each radius, CLUMPFIND identifies a relatively narrow range of SFRs, set by the threshold for detection against the background (see Figure 11). Since CLUMPFIND does not perform a local background subtraction before measuring the radius and brightness of each clump, and above the set threshold all pixels are assigned to a clump, there is a tendency for faint clumps to be too bright and too large. This effect is evident in the GOALS systems because our HST near-infrared images are typically much deeper than the IFU data used to measure the high- $z$ starbursts, and the local images contain measurable diffuse ionized gas, which must be

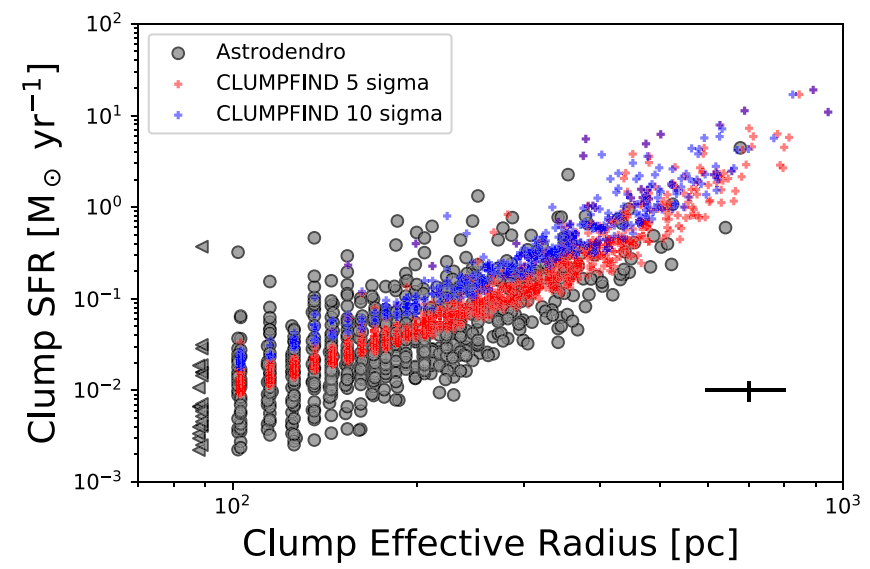

Figure 11. Direct comparison of the results of the CLUMPFIND and Astrodendro (see text for details) algorithms on 10 LIRGs from GOALS. Gray circles are clumps found using Astrodendro. Plus signs are clumps identified using CLUMPFIND using a $10 \sigma$ cutoff (in blue) and a $5 \sigma$ cutoff (in red). As discussed in the text, CLUMPFIND fails to find the full range in clump luminosities at a given radius. Bright clumps are shifted to larger radii, while faint clumps are boosted in luminosity.

subtracted to calculate the full range of clump properties, especially at the faint end.

\section{ORCID iDs}

K. L. Larson (iD https://orcid.org/0000-0003-3917-6460

T. Díaz-Santos (ib https://orcid.org/0000-0003-0699-6083

L. Armus (iD https://orcid.org/0000-0003-3498-2973

G. C. Privon (iD https://orcid.org/0000-0003-3474-1125

S. T. Linden (1D https://orcid.org/0000-0002-1000-6081

V. Charmandaris (i) https://orcid.org/0000-0002-2688-1956

V. U (1) https://orcid.org/0000-0002-1912-0024

D. B. Sanders (1D https://orcid.org/0000-0002-1233-9998

S. Stierwalt (ib https://orcid.org/0000-0002-2596-8531 
L. Barcos-Muñoz (1) https://orcid.org/0000-0003-0057-8892

J. Rich (i) https://orcid.org/0000-0002-5807-5078

A. Medling (1) https://orcid.org/0000-0001-7421-2944

D. Cook (10 https://orcid.org/0000-0002-6877-7655

A. Oklopĉić (1) https://orcid.org/0000-0002-9584-6476

E. J. Murphy (i) https://orcid.org/0000-0001-7089-7325

\section{References}

Alonso-Herrero, A., Rieke, G. H., Rieke, M. J., \& Scoville, N. Z. 2000, ApJ, 532,845

Alonso-Herrero, A., Rieke, G. H., Rieke, M. J., et al. 2006, ApJ, 650, 835

Armus, L., Heckman, T. M., \& Miley, G. K. 1989, ApJ, 347, 727

Armus, L., Mazzarella, J. M., Evans, A. S., et al. 2009, PASP, 121, 559

Cava, A., Schaerer, D., Richard, J., et al. 2018, NatAs, 2, 76

Chandar, R., Fall, S. M., \& Whitmore, B. C. 2015, ApJ, 810, 1

Cook, D. O., Dale, D. A., Lee, J. C., et al. 2016, MNRAS, 462, 3766

Cosens, M., Wright, S. A., Mieda, E., et al. 2018, ApJ, 869, 11

Cowie, L. L., Hu, E. M., \& Songaila, A. 1995, AJ, 110, 1576

Daddi, E., Alexander, D. M., Dickinson, M., et al. 2007, ApJ, 670, 156

Daddi, E., Elbaz, D., Walter, F., et al. 2010, ApJL, 714, 118

de Grijs, R., Anders, P., Bastian, N., et al. 2003, MNRAS, 343, 1285

Dekel, A., Sari, R., \& Ceverino, D. 2009, ApJ., 703, 785

Díaz-Santos, T., Charmandaris, V., Armus, L., et al. 2011, ApJ, 741, 32

Elbaz, D., Daddi, E., Le Borgne, D., et al. 2007, A\&A, 468, 33

Elbaz, D., Dickinson, M., Hwang, H. S., et al. 2011, A\&A, 533, 119

Elmegreen, B. D., Elmegreen, D. M., \& Hirst, A. C. 2004, ApJ, 612, 191

Elmegreen, D. M., Elmegreen, B. G., Marcus, M. T., et al. 2009, ApJ, 701, 306

Feldmann, R., Hopkins, P. F., Quataert, E., et al. 2016, MNRAS, 458, 14

Fisher, D. B., Glazebrook, K., Damjanov, I., et al. 2017, MNRAS, 464, 491

Förster Schreiber, N. M., Shapley, A. E., Genzel, R., et al. 2011, ApJ, 739, 45

García-Marín, M., Colina, L., \& Arribas, S. 2009, A\&A, 505, 1017

Genzel, R., Burkert, A., Bouché, N., et al. 2008, ApJ, 687, 59

Genzel, R., Newman, S., Jones, T., et al. 2011, ApJ, 733, 101

Guo, Y., Rafelski, M., Bell, E. F., et al. 2018, ApJ, 853, 108
Jones, T. A., Swinbank, A. M., Ellis, R. S., et al. 2010, MNRAS, 404, 1247 Kennicutt, R. C., Jr. 1998, ApJ, 498, 541

Kennicutt, R. C., Jr., \& Evans, N. J. 2012, ARA\&A, 50, 531

Kroupa, P. 2001, MNRAS, 322, 231

Larson, K. L., Sanders, D. B., Barnes, J. E., et al. 2016, ApJ, 825, 128

Leitherer, C., Schaerer, D., Goldader, J. D., et al. 1999, ApJS, 123, 3

Leroy, A. K., Walter, F., Brinks, E., et al. 2008, AJ, 136, 2782

Linden, S. T., Song, Y., Evans, A. S., et al. 2019, ApJ, 881, 70

Livermore, R.C., Jones, T., Richard, J., et al. 2012, MNRAS, 427, 688

Livermore, R.C., Jones, T., Richard, J., et al. 2015, MNRAS, 450, 1812

Maíz Apellániz, J., \& Úbeda, L. 2005, ApJ, 626, 873

Maragkoudakis, A., Zezas, A., Ashby, M. L. N., \& Willner, S. P. 2017, MNRAS, 466, 1192

Mieda, E., Wright, S., Larkin, J. E., et al. 2016, ApJ, 831, 78

Murphy, E. J., Condon, J. J., Schinnerer, E., et al. 2011, ApJ, 737, 67

Nguyen-Luong, Q., Nguyen, H. V. V., Motte, F., et al. 2016, ApJ, 833, 23

Oklopčić, A., Hopkins, P. F., Feldmann, R., et al. 2017, MNRAS, 465, 952

Pereira-Santaella, M., Colina, L., García-Burillo, S., et al. 2016, A\&A, 587, 44

Petric, A. O., Armus, L., Howell, J., et al. 2011, ApJ, 730, 28

Piqueras López, J., Colina, L., Arribas, S., et al. 2016, A\&A, 590, 67

Piqueras López, J., Colina, L., Arribas, S., \& Alonso-Herrero, A. 2013, A\&A, 553,85

Rodighiero, G., Daddi, E., Baronchelli, I., et al. 2011, ApJL, 739, L40

Sanders, D. B., Mazzarella, J. M., Kim, D.-C., et al. 2003, AJ, 126, 1607

Sanders, D. B., \& Mirabel, I. F. 1996, ARA\&A, 34, 749

Schmidt, M. 1959, ApJ, 129, 243

Soto, E., de Mello, D. F., Rafelski, M., et al. 2017, ApJ, 837, 6

Stierwalt, S., Armus, L., Charmandaris, V., et al. 2014, ApJ, 790, 124

Stierwalt, S., Armus, L., Surace, J. A., et al. 2013, ApJS, 206, 1

Swinbank, A. M., Smail, I., Sobral, D., et al. 2012, ApJ, 760, 130

Tacconi, L. J., Genzel, R., Smail, I., et al. 2008, ApJ, 680, 246

Tamburello, V., Mayer, L., Shen, S., \& Wadsley, J. 2015, MNRAS, 453, 2490

U, V., Medling, A. M., Imami, H., et al. 2019, ApJ, 871, 166

Williams, J., De Geus, E. J., \& Blitz, L. 1994, ApJ, 428, 693

Wisnioski, E., Glazebrook, K., Blake, C., et al. 2012, MNRAS, 422, 3339

Zhang, Q., \& Fall, S. M. 1999, ApJL, 527, L81 